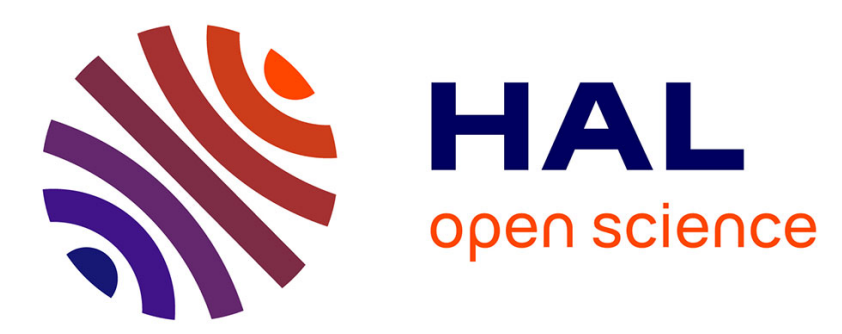

\title{
Characterisation and modeling of a pulsed molecular beam
}

\author{
A. Lietard, G. Gallician, J. Tan, M.-A. Gaveau, M. Briant, B. Soep, J.-M. \\ Mestdagh, L. Poisson
}

\section{- To cite this version:}

A. Lietard, G. Gallician, J. Tan, M.-A. Gaveau, M. Briant, et al.. Characterisation and modeling of a pulsed molecular beam. Molecular Physics, 2021, 119, pp.e1737743. 10.1080/00268976.2020.1737743 . cea-03053669

\section{HAL Id: cea-03053669 https://hal-cea.archives-ouvertes.fr/cea-03053669}

Submitted on 24 Nov 2021

HAL is a multi-disciplinary open access archive for the deposit and dissemination of scientific research documents, whether they are published or not. The documents may come from teaching and research institutions in France or abroad, or from public or private research centers.
L'archive ouverte pluridisciplinaire HAL, est destinée au dépôt et à la diffusion de documents scientifiques de niveau recherche, publiés ou non, émanant des établissements d'enseignement et de recherche français ou étrangers, des laboratoires publics ou privés. 


\title{
ARTICLE
}

\section{Characterization and Modeling of a Pulsed Molecular Beam}

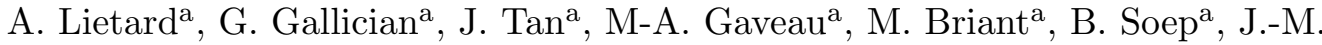 \\ Mestdagh $^{\mathrm{a}}$, L. Poisson ${ }^{\mathrm{a}}$ \\ ${ }^{a}$ Université Paris-Saclay, CEA, CNRS, LIDYL, 91191, Gif-sur-Yvette, France.
}

\section{ARTICLE HISTORY}

Compiled February 27, 2020

\begin{abstract}
Standard techniques used in real-time reaction dynamics experiments, namely photoionization by a pulsed laser and velocity imaging are applied to document the time dependent velocity distribution of 2-hydroxypyridine (2-HP) molecules in a pulsed beam where 2-HP is seeded in either helium or argon. The purpose is to identify features which cannot be assigned to a pure free molecular (effusive in the present case) or a pure continuum (supersonic here) flow regime. The beam is generated in a two stage expansion, where the first stage is driven by a pulsed valve. The experimental work is complemented by simulations. Two phenomena retain the attention: i) a slow return to the effusive flow regime after the valve opening has generated an intense supersonic flow; ii) development of a shock wave a short time after the valve opening.
\end{abstract}

\section{KEYWORDS}

\section{Introduction}

Studies in fundamental physical chemistry often require that the species of interest are carried in molecular beams, an ideal isolation environment for investigating intrinsic properties of these species. Supersonic expansion is the dedicated technique to generate intense molecular beams having a narrow velocity distribution.[1] To be efficient, these expansions must proceed from a high gas pressure ranging from a few bars up to tens of bars. [2-4] This is especially easy to achieve when expanding rare gases and therefore, the species of interest must be mixed with a rare gas carrier before the expansion, or seeded into the carrier gas during the expansion. In most cases, especially when the species of interest is solid or liquid at room temperature, the seeding technique prevails and various systems have been designed. They differ whether the species of interest has a substantial vapor pressure at room temperature or need to be vaporized.

Several methods are used to seed the species of interest during the expansion of the carrier gas. Accordingly, the species of interest is brought collisionally into the beam at a location where the flow regime may not be purely supersonic. [3] This point has attracted the attention very early, especially because the expansion dynamics which are at play, are believed to be responsible for the rotational alignment of the seeded species. $[5,6]$. 
The collisionally driven motion of seeded species can also exhibit a time-dependent velocity distribution when the supersonic expansion of the carrier gas is actuated by a pulsed valve. This point has attracted our interest in a purely experimental work where tools originally developed for real-time reaction dynamics studies were used to characterize a time dependant flow regime in a pulsed supersonic expansion. [7]

The points above have motivated the present work, which is both experimental and computational. On the experimental side, 2-hydroxypyridine ( pyridin-2-ol, CAS No.: 142-08-5, hereafter labeled 2-HP) is seeded into a pulsed beam of helium (or argon) and, as in our early publication, tools usually used for running femtosecond pump-probe experiments are used for monitoring the time dependent velocity distribution of 2-HP within the beam.

On the computational side, a model is developed where the continuum and free molecular regimes of the expansion are described analytically, whereas the transition between these two regimes is treated by an empirical switch function. The resulting simulations are used to help discussing the experimental results. A full theoretical treatment of the expansion is beyond the scope of the present work. In particular, the Direct Simulation Monte Carlo (DSMC) method, first introduced by Bird in the 60's[8] could be used to simulate this unsteady flow from the continuous to the molecular regime. The idea here is to motivate such a treatment by unraveling salient effects of the transition regime during the gas expansion.

\section{Experimental setup}

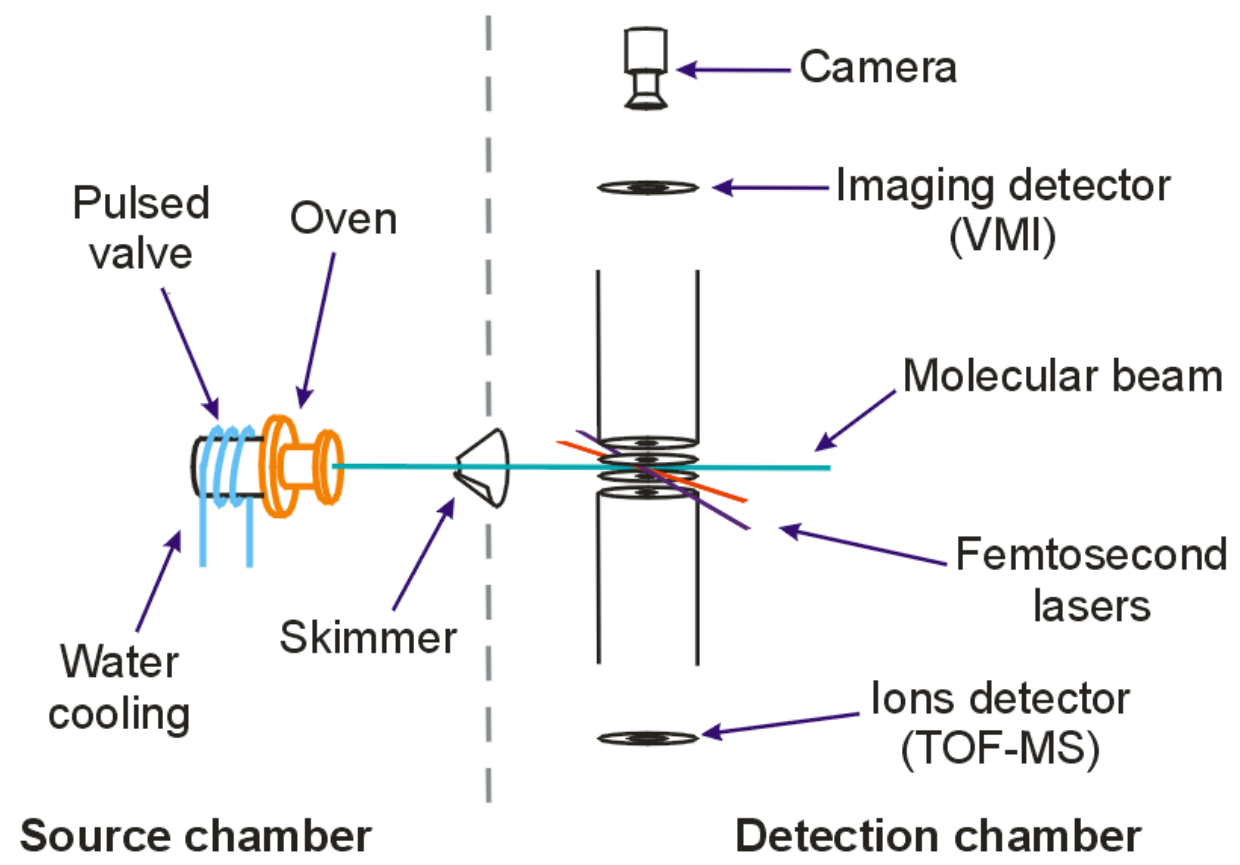

Figure 1. Experimental setup. In the present paper, the Time-of-Flight Mass Spectrometer (TOF-MS) is not used.

Our laboratory is equipped with several Velocity Map Imaging (VMI) detectors for documenting the angular and velocity distribution of photoions and photoelectrons 
in real-time reaction dynamics studies. [9] More precisely, the present experimental setup is designed to inform on the velocity and the angular distribution of reaction products in a pump-probe femtosecond laser arrangement where the pump pulse carries the system under study on an excited potential energy surface and the probe pulse monitors the resulting dynamics as a function of the pump/probe delay. It operates by photoionization. [10, 11]

The present paper offers an unusual use of a VMI detector. It is used to provide information on the transient molecular flow generated by a pulse valve. Accordingly, a single laser pulse is used to document the velocity and angular distribution of the seeded species 2-HP within the pulsed rare gas (helium or argon) beam generated by the beam source. The time dependence which is analysed here is that between the valve opening and the laser pulse. Other examples exist in the literature where a VMI is used to characterize a pulsed molecular beam source (e.g. Ref. [12]).

The full setup is shown in Fig. 1. It associates a source chamber which generates a pulsed molecular beam where 2-HP is carried into helium or argon and a detection chamber where the molecular beam crosses the probe laser. The pulse valve is manufactured by First Sensor valve (formerly General Valve), and beyonds to the Serie 9. It can afford up to 86 bars and its minimal opening time is $160 \mu \mathrm{s}$. Photoions (or photoelectrons) formed by the beam/probe interaction are extracted by two voltages, the repeller and extractor voltages, in a perpendicular direction to the beam. The distances between each electrodes was fixed at $16 \mathrm{~mm}$. The length of the flying tube is $465 \mathrm{~mm}$. In the current experiments, the voltages were set at $2000 \mathrm{~V}$ for the repeller and $1441 \mathrm{~V}$ for the extractor. Here, photoions are detected but when reversing the voltages, photoelectrons can be extracted as well (see below). These voltages are associated within a Velocity Map Imaging (VMI) detector. The latter produces images where the angular and radial distribution of the photoion signal represents the angular and velocity distribution of the 2-HP molecules within the beam.[9] The detector is a $80 \mathrm{~mm}$ diameter chevron of MCPs coupled to a P20 phosphor screen furnished by Photonis Scientific, Inc. The voltage applied to the MCPs is the superposition of a bias voltage of $1200 \mathrm{~V}$ with a pulse gate voltage of $600 \mathrm{~V}$, which opening time and duration are tunable to gate a specific mass. A PCO1600s camera (from PCO) has been used which is a 14 bits cooled CCD Camera.

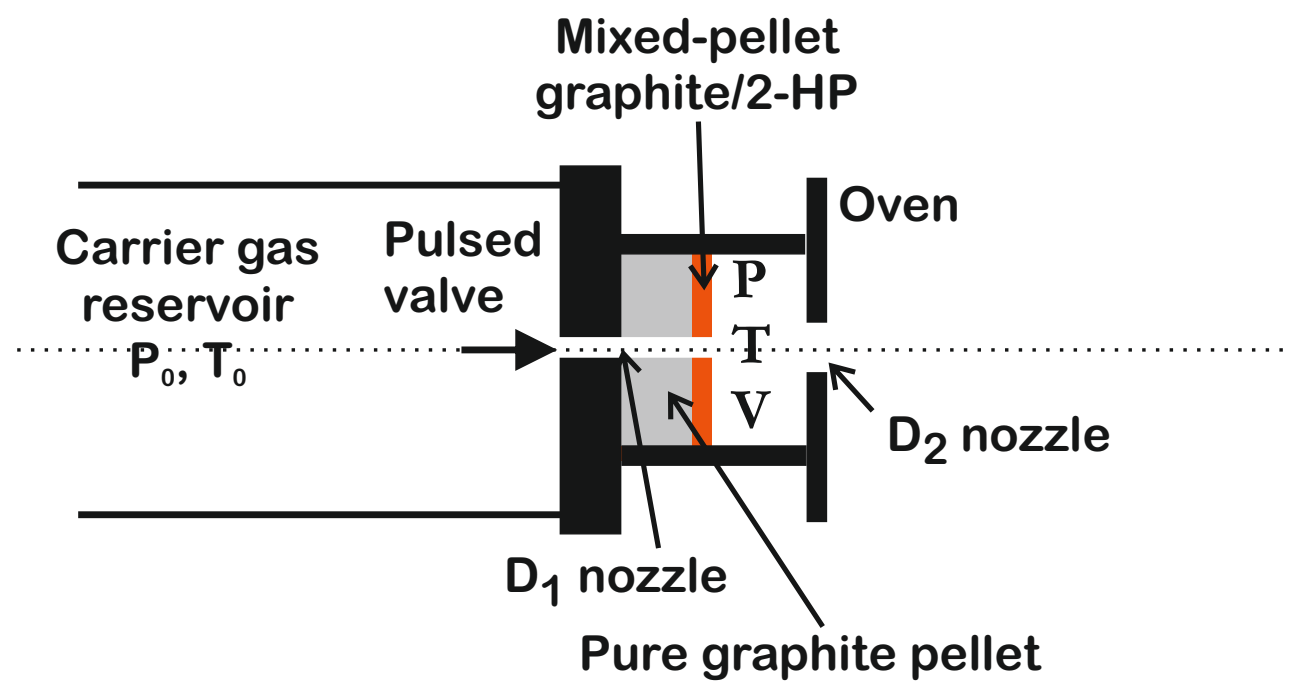

Figure 2. Not to scale scheme of the beam source (Pulsed valve + Oven). 
The beam source (Pulse valve + Oven in Fig. 1) offers a clear separation between the initial supersonic expansion of the carrier gas and the seeding zone. An expanded view is shown in Fig. 2. It was first designed by Shafizadeh et al.[13]. The vaporization of 2-HP proceeds from a pellet which is obtained by pressing together 2-HP and graphite powders. The resulting mixed-pellet is mounted on a pure graphite pellet on the exit flange of a pulse valve operating with pure carrier gas (helium or argon) at a $20 \mathrm{~Hz}$ repetition rate. The pellets are heated at the desired temperature by a resistive heater. Here, the vapor pressure of 2 -HP is large enough $\left(P_{s} \sim 3.410^{-4}\right.$ mbar at $\left.25^{\circ} \mathrm{C}[14]\right)$ to operate the oven at room temperature. The pellets are drilled with a $2 \mathrm{~mm}$ hole to the carrier gas to expand freely.

Two sequential expansions take place in the beam source. The first one proceeds from the pulsed valve and goes from the Rare Gas Stagnation Chamber (stagnation conditions $P_{0}, T_{0}=$ room temperature) to the oven through a nozzle of diameter $D_{1}=$ $300 \mu \mathrm{m}$. The second expansion proceeds through a secondary nozzle (diameter $D_{2}=$ $2.0 \mathrm{~mm}$ ) from the oven to the source chamber where a low pressure $P_{1} \sim 10^{-5}$ mbar is maintained by turbo pumps. Helium and argon are used as carrier gas. Their stagnation pressure $P_{0}$ is chosen between 4 and 9 bar.

After extraction by a conical skimmer (diameter $d_{e}=1 \mathrm{~mm}$, located at a $l=5 \mathrm{~cm}$ distance from the $D_{2}$ nozzle), the molecular beam enters into the detection chamber $\left(P_{2} \sim 10^{-7}\right.$ mbar) where it crosses perpendicularly a femtosecond laser beam (LUCA part of the SLIC European facility).[11] The laser wavelength is the third harmonic of a Ti:Saphire laser. It is measured at $265 \mathrm{~nm}(4.69 \mathrm{eV})$ with a $\sim 3 \mathrm{~nm}$ full-width-athalf-maximum. It ionizes the 2-HP molecules (8.933 eV ionization)[15] in a resonant two-photon process[16]. Of course, the present experiment could be performed using a nanosecond laser but as said above, existing tools of a femtochemistry setup are used here.

The crossing zone between the beam and the laser is located at a distance $R=$ $17.5 \mathrm{~cm}$ from the $D_{2}$ nozzle (exit orifice of the oven).

\section{Experimental Results}

An experiment consists in recording a series of photoion images (1200 pixels $\times 1200$ pixels) as a function of the time delay between the pulsed valve opening and the laser pulse. Each image is accumulated for $c a .20$ laser pulses.

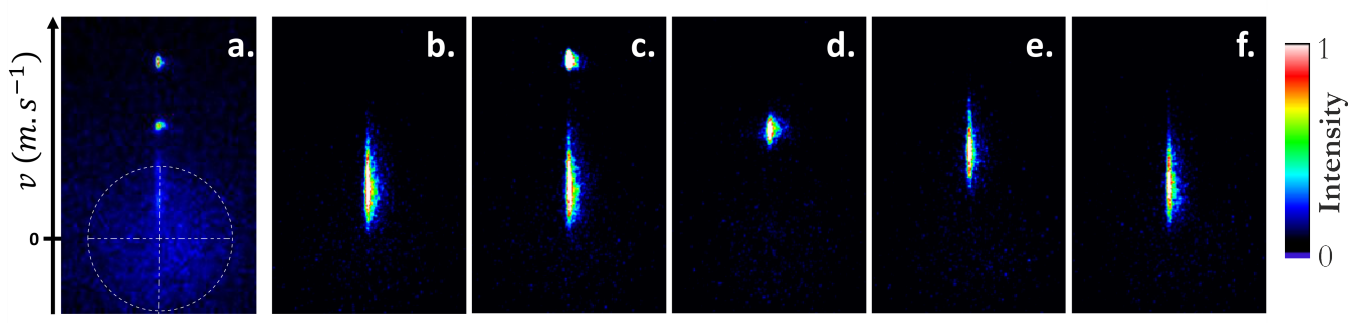

Figure 3. Photoion images. The beam is generated by a 6 bar expansion in argon. Image a) is not massgated and shows the thermal distribution of the background gas. The cross centre marks the origin of velocities. Images b) to f) are gated to the 2-HP mass. The time delay between the valve opening and the detection laser pulse increases from b) to f) respectively $0.23 \mathrm{~ms}, 0.38 \mathrm{~ms}, 1.03 \mathrm{~ms}, 5.68 \mathrm{~ms}$ and $38.48 \mathrm{~ms}$ from the pulsed valve opening time. The beam is directed along the vertical axis of the images.

Typical photoion images are shown in Fig. 3. The halo in image a) is associated 
with the multiphotonic ionization of the residual gas. The centre of the observed halo indicates the zero velocity. It is represented by a cross in the figure. In the other images (b-f), the intense elongated signal is due to the ionization of 2-HP in the beam. It shows the gas flow as it flies through the detection zone.

The images are aligned with the beam axis along their vertical axis. The larger the distance between the 2-HP signal and the zero velocity centre, the larger the velocity of 2-HP along the beam axis. A calibration is needed to relate this distance to the velocity of 2-HP. Note that only velocities along the beam axis are relevant here since velocities along the perpendicular directions are limited by the beam collimation.

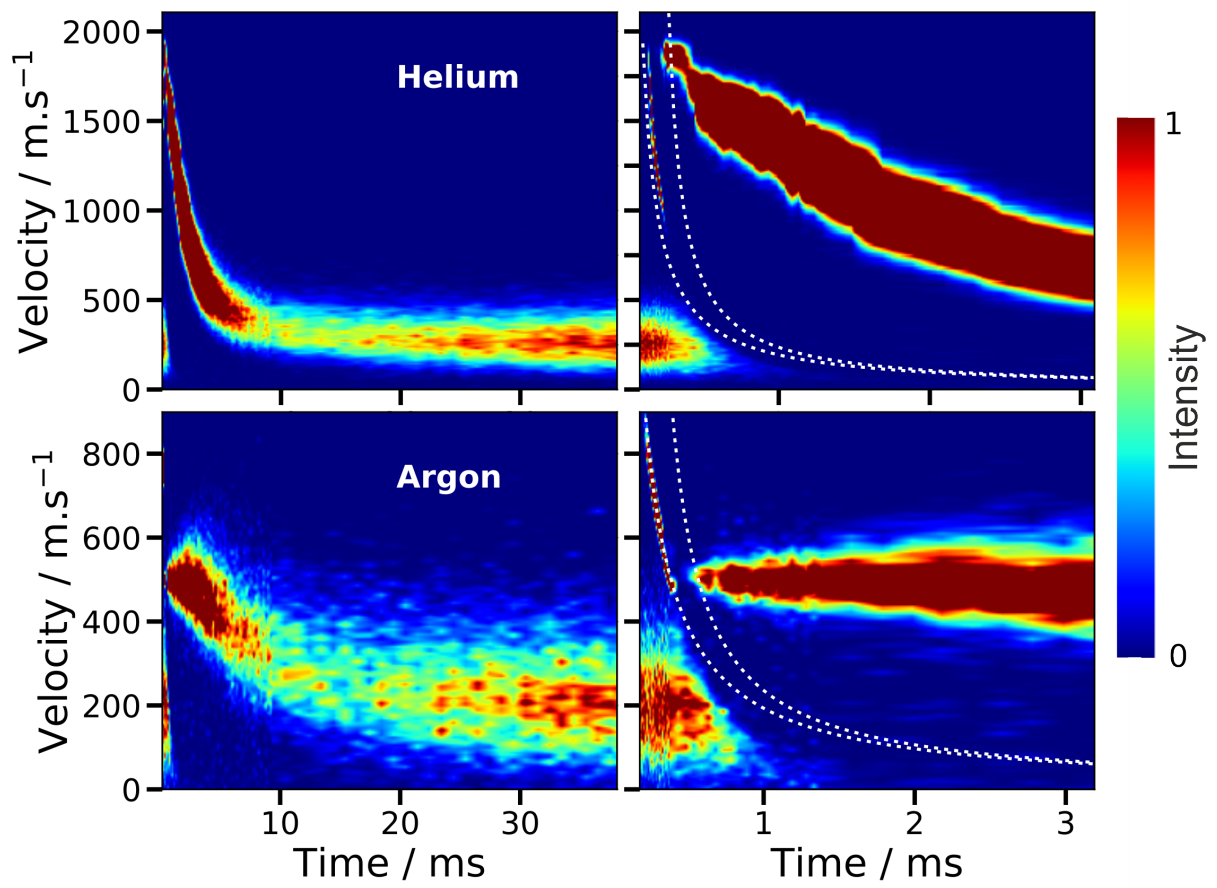

Figure 4. Intensity (colour scale) of the 2-hydroxypyridine (2-HP) photoions as a function of the longitudinal photoion velocity (vertical scale in $\mathrm{m} . \mathrm{s}^{-1}$ ) and delay time between the valve opening and the laser pulse (horizontal scale in ms). The upper (resp. lower) panels refer to a $\mathrm{P}_{0}=6$ bar expansion of helium (resp. argon). The left (resp. right) hand-side panels show the evolution over $30 \mathrm{~ms}$ (resp. $3 \mathrm{~ms}$ ). The white dotted curves

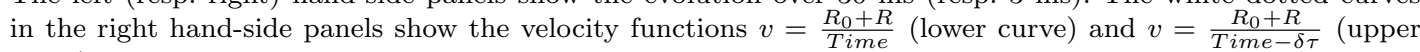
curve), $R_{0}=1.83 \mathrm{~cm}$ being the distance between the $D_{1}$ and $D_{2}$ nozzles, $R=17.5 \mathrm{~cm}$ the distance between the $D_{2}$ nozzle and the detection zone and $\delta \tau=180 \mu$ s the opening time of the valve.

The necessary distance-to-velocity calibration takes advantage of the possibility mentioned above. The VMI can be switched from photoion to photoelectron detection by simply reversing the repeller and extractor voltages. Hence, the calibration does not change and the well-known ionization energetics of $\mathrm{O}_{2}$ can be used to establish the correspondance between the number of pixels from the image centre and the kinetic energy of the detected particle.[17] The following factor is obtained for the pixel $\rightarrow e V$ correspondence: $\left(\frac{E x t}{R e p}-0.51\right) \times(\operatorname{Rep}+77.0) \times 3.93 \times 10^{-7}$, where Ext and Rep are the extractor and repeller voltages, respectively. Finally, the kinetic energy is converted in velocity as $v=\sqrt{\frac{2 e E}{m_{p}}}$, where $e$ is the electron charge and $m_{p}$ the mass of 2-HP (95.10 u).

After performing the pixel $\rightarrow \mathrm{eV}$ conversion on each image, a series of curves showing the photoion intensity as a function of the photoion velocity along the beam 
axis is obtained. By bringing these curves together, a figure can be constructed where the photoion intensity (color scale) is shown as a function of the photoion velocity (vertical scale) and the time delay (horizontal scale) between the valve opening and the laser pulse.

Fig. 4 reports the corresponding results

for the two carriers gases, helium (upper line) and argon (bottom line). In both cases, the stagnation pressure $\mathrm{P}_{0}$ is 6 bar and the opening time of the valve is $\delta \tau=180 \mu \mathrm{s}$. Time zero in Fig. 4 is chosen to correspond to the valve opening and is corrected for the dead-time of the valve controller $(0.32 \mathrm{~ms})$. The time dependencies shown in Fig. 4 are followed up to $38 \mathrm{~ms}$ (left column). The first $3.1 \mathrm{~ms}$ are zoomed in the right column of the figure.

Other experiments were run at $\mathrm{P}_{0}=4,6$ and 9 bar with argon as carrier gas. A different sampling is used as a function of time to get finer details on the first $3.1 \mathrm{~ms}$ evolution of the signals. The corresponding signals are shown in Fig. 5. For these experiments, the opening time of the valve is $\delta \tau=190 \mu \mathrm{s}$.

Three regions can be distinguished in Figs. 4 and 5:

i) A small velocity region - A velocity class centred about $200 \mathrm{~m} \cdot \mathrm{s}^{-1}$ is present at the beginning of the time dependent behavior, for less than $0.3 \mathrm{~ms}$ after the valve opening, and reappears after about $20 \mathrm{~ms}$.

ii) An intermediate region - A complex region exists between 0.3 and $0.5 \mathrm{~ms}$ in helium (0.5-0.7 $\mathrm{ms}$ in argon) where three classes of velocities coexist at the same delay time.

iii) A decreasing velocity region - The intermediate region is followed by an extended one where the velocity varies over a wide range, from $1600 \mathrm{~m} . \mathrm{s}^{-1}$ at $\approx 0.5 \mathrm{~ms}$ in helium (resp. $550 \mathrm{~m} \cdot \mathrm{s}^{-1}$ at $\approx 1 \mathrm{~ms}$ in argon) down to the low velocity class $\left(\approx 200 \mathrm{~m} \cdot \mathrm{s}^{-1}\right)$.

\section{Discussion of the experimental results}

The valve opening (time zero in Figs. 4 and 5) creates a gas pulse within the beam source, which has to cover a $17.5 \mathrm{~cm}$ distance before reaching the detection region. The white curves in the right panels of Fig. 4 gives the time needed to cover this distance whether the gas particle leaves the oven at the opening (top curve) or closing (lower curve) of the valve. Events, which appear below the lower curve, happen prior the valve opens. Those happening after the valve is closed, appear above the top curve.

Clearly, the sudden increase of the detected velocities that appears in Fig. 4 near $0.5 \mathrm{~ms}$ in both the helium and argon expansions (point ii) above) is associated with a drastic change of the flow regime when the valve opens. In contrast, the signal with a velocity at about $200 \mathrm{~m} \cdot \mathrm{s}^{-1}$ which is observed at shorter delays corresponds to molecules coming from the oven before the valve opens. The fact that this velocity component disappears after $0.5 \mathrm{~ms}$ and reappears at much longer time (see the observation point $i$ ) in the section above) is an indication that the flow regime which exists prior the valve opening is recovered after the valve has closed when the carrier gas is totally pumped out. This picture has been used to build the three step scenario schemed in Figure 6: 

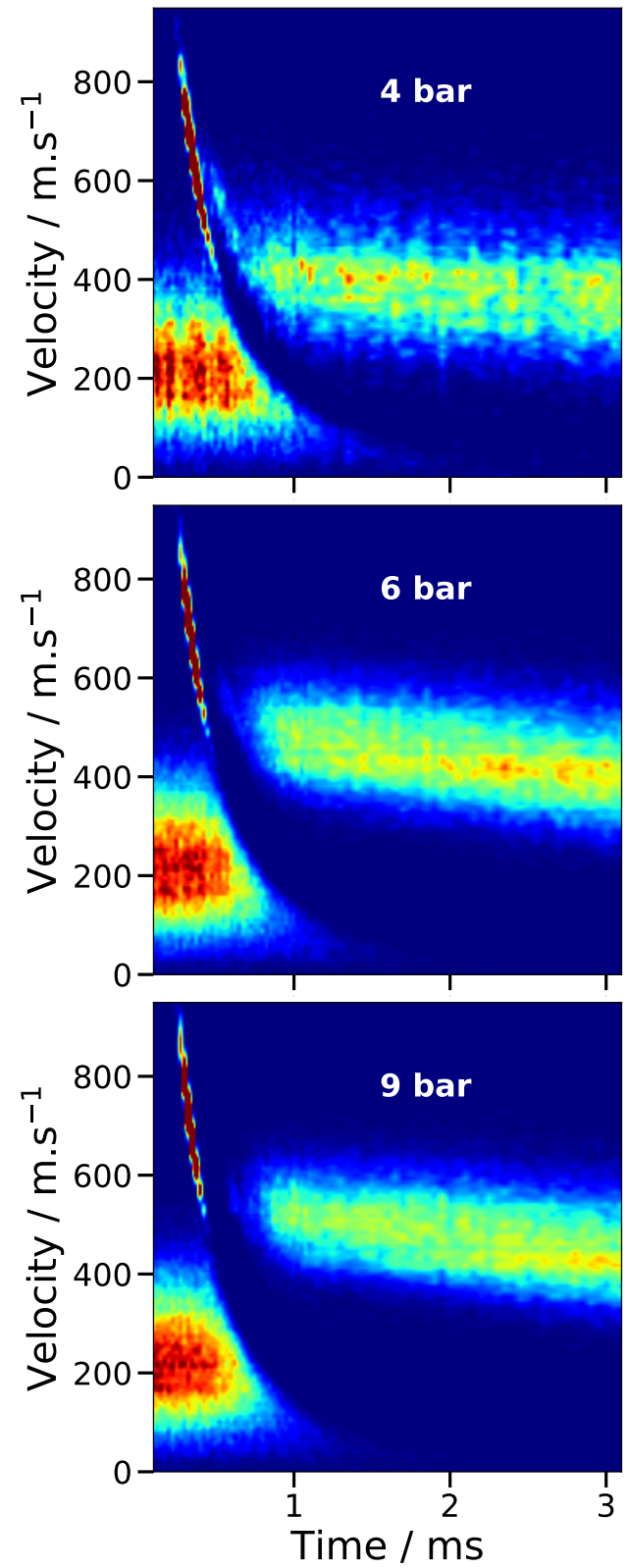

Figure 5. Experimental 2-HP ion signal as a function of the 2-HP velocity and delay time between the valve opening and laser pulse. The carrier gas is argon. The stagnation pressure $P_{0}$ labels each panel. It shows that the velocities of the molecules are rising with the stagnation pressure, whereas the velocity distribution sharpens. The color scale is the same than that of Fig. 4. 

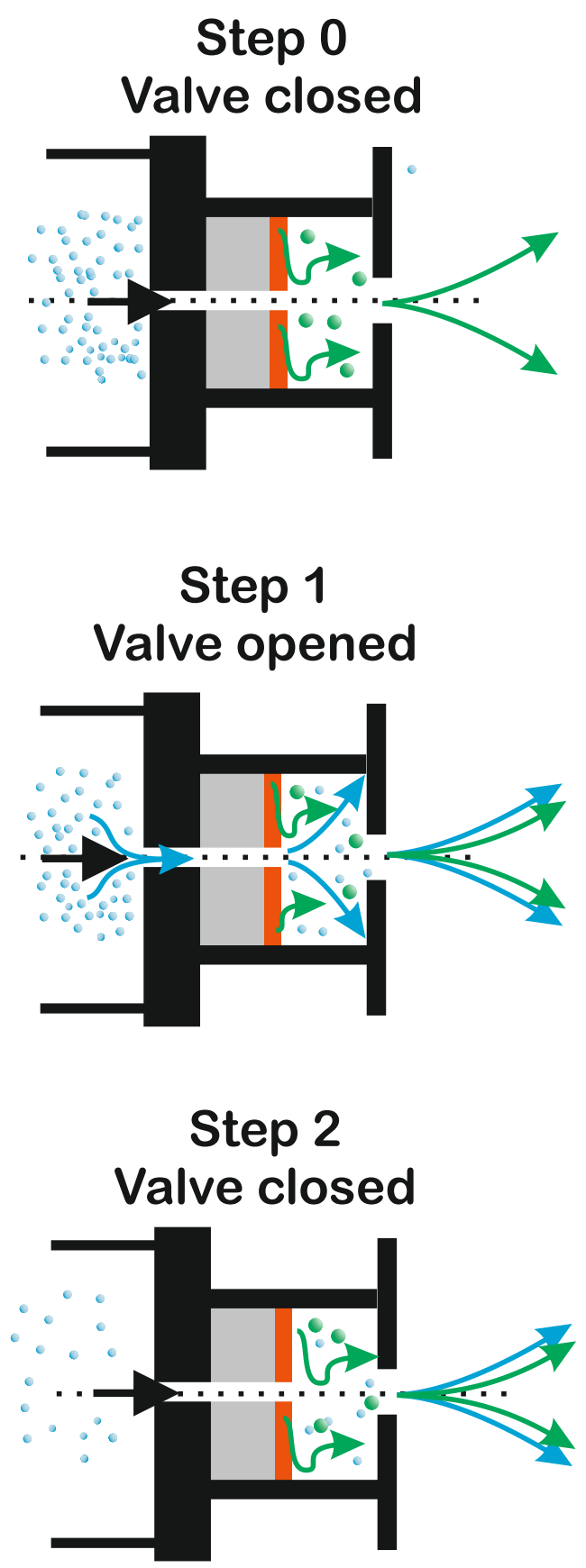

Figure 6. Scheme showing the flow of 2-HP molecules (in green) and carrier gas atoms (in blue) entering and leaving the oven at each cycle of the pulse valve. 
Step 0: The valve is closed and the carrier gas from the previous valve opening is fully pumped out. The valve is pulsed at a $20 \mathrm{~Hz}$ repetition rate (period $T_{r}=50 \mathrm{~ms}$ ) and its opening time $\delta \tau$ of less than $200 \mu$ s lets enough time to the carrier gas to be pumped out totally between two successive valve openings. The only gas present at this step is therefore 2-HP molecules which evaporate from the pellet and generate a vapor in the oven. The latter expands into the source chamber through the nozzle of diameter $D_{2}$ which is open all the time. A steady state regime is at play where the expansion is likely effusive and responsible for the velocity class centred at $c a$. $200 \mathrm{~m} \cdot \mathrm{s}^{-1}$.

Step 1: When the valve opens up, the carrier gas fills the oven through the valve nozzle (diameter $D_{1}$ ) via a first supersonic expansion. The pressure inside the oven increases sharply as a function of time. This generates a gas flow to the source chamber through the output orifice of the oven (nozzle of diameter $D_{2}$ ). A continuum flow regime where many carrier gas/2-HP collisions are present, is building up during this step, which stops when the valve shuts down at time $\delta \tau$. If the gas pressure rises enough in the oven, this secondary expansion can be supersonic. This step appears between the two white curves drawn in the right column of Fig. 4. It coincides with the complex region mentioned at point ii) in the section above. The flow regime which is turned on by the valve opening replaces completely that at play at step 0 .

Step 2: When the valve closes, the oven starts emptying towards the source chamber through the $D_{2}$ nozzle, which is always opened. The output flow decreases gradually down and switches to a free molecular flow when the pressure in the oven becomes low enough. Such change in the flow regime might be the origin of the substantial velocity decrease mentioned at point iii) above. Observing Fig. 4 in detail, the decrease of the average velocity is associated with an enhancement of the width of the velocity distribution. This is a further indication that the flow regime is changing along this step.

Actually, step 0 is the end of step 2 and observation of Fig. 4 confirms the anticipation above that the time interval between two successive valve opening, $T_{r}=50 \mathrm{~ms}$, let enough time for the carrier gas to be fully pump out. After $30 \mathrm{~ms}$ indeed the flow regime is back to that encountered before the valve opening.

\section{Modeling the gas flow inside the beam source}

The notations used in this section are defined in the text when they first appear. They are listed all together in the appendix A. The value of the corresponding quantities is also given in the appendix.

\subsection{Nature of the flow regimes to be considered}

When discussing the three step operating mode of the beam source in the section above, we suggested that over an entire cycle of the pulsed valve, two flow regimes are experienced: a free molecular one when the gas expansion is effusive (step 0, identical with the end of step 2 and possibly beginning of step 1 when the carrier gas starts filling the oven); a continuum flow when the two sequential expansions are supersonic essentially (most of step 1 and beginning of step 2). Actually the transition between 
the molecular and the continuum flow regimes is unlikely sudden, especially during step 2 when the oven is slowly emptying. A transition regime must be considered between the two extreme flow regimes.

\subsection{Empirical switch function}

The integral-differential nature of the Boltzmann equation raises many mathematical difficulties when modeling gas flows by gas-kinetic algorithms when the flow covers continuum, slip, transitional and free molecular regimes (see review by Li et al.[18]

For not facing these difficulties we developed a phenomenological approach for treating of the transition between the free molecular and the continuum flow regimes, which prevail in the present experiment. Analytical expressions are derived below to describe the flow rate and the velocity distribution of 2-HP in these two extreme regimes and a single numerical function (hereafter called the switch function) is used to switch from those expression describing the free molecular regime to those describing the continuum regime.

The dimensionless Knudsen number $\left(K_{n}\right)$ is used for quantifying the switch between the extreme regimes. It is defined as the ratio $K_{n}=\frac{\lambda_{0}}{D}$ between the mean free path $\lambda_{0}$ of the gas particles and a characteristics length $D$ of the gas flow. In the present context, $D$ is the diameter of the nozzle through which the gas is expanding (i.e. $D_{1}$ or $D_{2}$ whether the first or the second expansion is considered). A free molecular flow is associated with a large Knudsen number $\left(K_{n} \gtrsim 1\right)$, whereas $K_{n} \ll 1$ characterizes a continuum flow. In the latter case, if the proper pressure conditions are satisfied, the expansion can be supersonic. This is essentially the case here. In practice, we shall see below that only the expansion through the $D_{2}$ nozzle need to be switched between the effusive and supersonic flow regimes using the Knudsen number.

Three different switch functions $f_{S}\left(K_{n}\right)$ were sampled. They are shown in Fig. 7. The first one, constructed with an arctangent function, mimics a smooth transition. We chose $\log \left(K_{n}\right)=-1$ as the limit between the two regimes. The second switch function is built on two half-parabola and reflects a fast transition. The slope is chosen to be the same in the transition between the two regimes, but the switch starts closer to this region. The third switch function is composite. It follows the fast one when $\log \left(K_{n}\right) \leq-1$ and the smooth one above.

The criterium chosen to select the best switch function is the comparison between the experimental results of Fig. 4 and the simulations performed using these functions. The best choice is the composite function. Its analytical form is:

$$
f_{S}\left(K_{n}\right)= \begin{cases}1 & \text { if } \log \left(K_{n}\right)<-2 \\ \frac{1}{2}-\frac{1}{2}\left[\log \left(K_{n}\right)+1\right]^{2}-\left[\log \left(K_{n}\right)+1\right] & \text { if }-2 \leq \log \left(K_{n}\right)<-1 \\ \frac{1}{2}-\frac{1}{\pi} \arctan \left(\pi\left[\log \left(K_{n}\right)+1\right]\right) & \text { if }-1 \leq \log \left(K_{n}\right) .\end{cases}
$$

\subsection{Analytical description of the flow rate in the two extreme flow regimes}

We saw above that two extreme expansion flow regime are encountered, whether the value of $\log \left(K_{n}\right)$ is small ( $<-2$; supersonic expansion) or large ( $\geq 0$; effusive expansion). Gaveau [19] derived general expressions to describe the flow rate (number of particle 


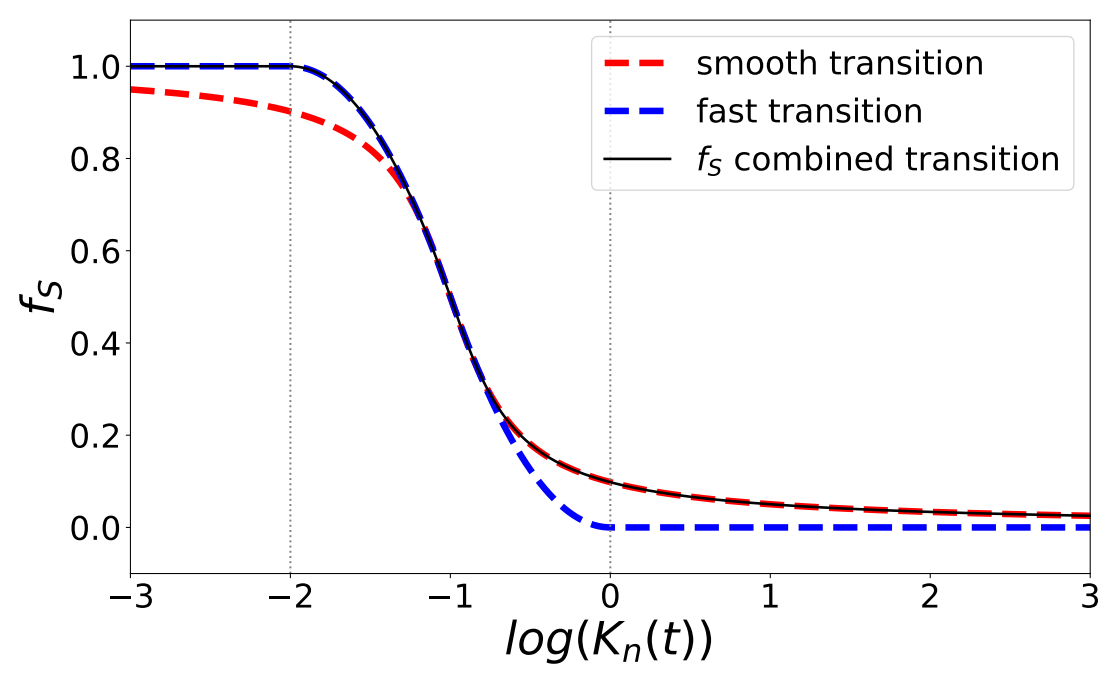

Figure 7. Functions used to switch between a molecular $\left(\log \left(K_{n}\right)>-0.3\right)$ and a continuum $\left(\log \left(K_{n}\right)<-1.5\right)$ flow regime.

per second) in both expansion regimes, assuming that an ideal gas is expanding.

The flow rate $\Phi_{\text {super }}$ of a supersonic expansion through a nozzle of diameter D is given by:

$$
\Phi_{\text {super }}(P)=P \frac{\pi}{4} \frac{\aleph_{A} D^{2}}{M} \sqrt{\frac{M}{\Re T_{0}} \gamma\left(\frac{2}{\gamma+1}\right)^{\frac{\gamma+1}{\gamma-1}}}
$$

whereas the flow rate $\Phi_{e f f}$ in an effusive expansion is given by:

$$
\Phi_{e f f}(P)=P \frac{\pi}{4} \frac{\aleph_{A} D^{2}}{M} \sqrt{\frac{1}{2 \pi} \frac{M}{\Re T_{0}}} .
$$

In these expressions, $P$ and $T_{0}$ are the stagnation pressure and temperature of the expanding gas, whose molar mass and heat capacity ratio are $M$ and $\gamma=\frac{C_{p}}{C_{v}}$, respectively. $\aleph_{A}$ and $\Re$ are the Avogadro number and the ideal gas constant, respectively. $\Phi_{\text {super }}(P)$ and $\Phi_{\text {eff }}(P)$ are expressed as a function of $P$, the only quantity which may vary as a function of time when the pulsed valve is operating. Note that the stagnation temperature for both expansions is equal to the source temperature $T_{0}$. The carrier gas which has experienced the first expansion spends enough time indeed in the oven to be thermalized again at the source temperature.

When the expansion proceeds from a gas mixture, $M$ and $\gamma$ must be weighted by the molar fraction of each gas.[20]. This is the case here with the second expansion which proceed from a mixture between the carrier gas and 2-HP molecules.

The only difference between Expressions 2 and 3 is a proportionality factor: $C=$ $\sqrt{\gamma\left(\frac{2}{\gamma+1}\right)^{\frac{\gamma+1}{\gamma-1}}}$ in the supersonic expansion (Eq. 2) which becomes $\sqrt{\frac{1}{2 \pi}}$ when the expansion is effusive (Eq. 3).

The flow rate $\Phi_{\text {trans }}(P)$ of the transition regime is modeled as a linear combination 
of $\Phi_{\text {super }}(P)$ and $\Phi_{\text {eff }}(P)$, weighted by the switch function $f_{S}\left(K_{n}\right)$ :

$$
\Phi_{\text {trans }}(P)=f_{S}\left(K_{n}\right) \cdot \Phi_{\text {super }}(P)+\quad\left[1-f_{S}\left(K_{n}\right)\right] \cdot \Phi_{\text {eff }}(P) .
$$

To get the value of $f_{S}\left(K_{n}\right)$ which appears in this expression, the Knudsen number $K_{n}=\frac{\lambda_{0}}{D_{2}}$ and therefore the mean free path

$$
\lambda_{0}=\frac{k_{B} T_{0}}{\sqrt{2} P \sigma}
$$

must be calculated. The quantities $k_{B}=\frac{\Re}{\aleph_{A}}$ and $\sigma$ in the latter expression are the Boltzmann constant and the elastic collision cross section between two carrier gas atoms, respectively. The latter choice tells that the Knudsen number is governed by the sole carrier gas. This approximation appears justified because the elastic collision cross-sections between two 2-HP molecules, between a 2-HP molecule and carrier gas atom and between two carrier gas atoms should not be dramatically different from one another. The elastic collision cross section between two helium (or argon) atoms is estimated as $\sigma=\pi\left(2 R_{v d w}\right)^{2}$ where $R_{v d w}$ is the relevant van der Waals radius.[21] Eq. 5 makes the Knudsen number an implicit function of the expanding gas pressure P. This will appear below in Eq. 18 at the very end of Sec. 5.6.

\subsection{Simulation of the flow rate out of the beam source}

Let us see how the above expressions are included in our own simulation software to evaluate the flow rate out of the oven along the three steps detailed in Sec. 4.

Step 0: effusive expansion of 2-HP. The valve is closed at this step and a steady state is reached where the evaporation of 2-HP molecules out of the mixed 2-hydroxypyridine/graphite pellet is balanced with the effusive expansion of these molecules through the nozzle of diameter $D_{2}$. Accordingly, the number of 2-HP molecules which leaves the surface of the pellet per unit of time, $\Phi_{m, i}$, is equal to flow rate of 2-HP molecules out of the oven, $\Phi_{m, o}$ (hereafter, subscript $m$ is a shortcut for $2-H P$ molecule).

$\Phi_{m, i}$ is controlled by the equilibrium between condensed molecules in the pellet and sublimated ones in the oven. Barret and Clement described such a situation in general terms when considering evaporation and condensation at surfaces as basic processes in which molecules transfer between a vapor and a solid condensate.[22] Eq. 22 of reference [22] for $\sigma^{*}=1$ (warning: in the work of Barrett and Clement[22], $\sigma^{*}$ is a free parameter, not a cross-section as in the present work) is used here for estimating the number of 2-HP molecules exiting the pellet surface per unit of time, assuming that pressure $p_{w}$ at the wall in the work of Barrett and Clement[22] is equal to the saturation vapor pressure $P_{s}$ of $2-\mathrm{HP}$ at the pellet temperature $T_{0}$. In the present context, the pressure $P_{m}$ of 2 -HP in the oven plays the role of pressure $p_{\infty}$ in the work by Barrett and Clement[22]. Accordingly, these assumptions lead to the Hertz-Knudsen formula:

$$
\Phi_{m, i}=\left(P_{s}-P_{m}\right) \frac{\aleph_{A} S_{p}}{M_{m}} \sqrt{\frac{1}{2 \pi} \frac{M_{m}}{\Re T_{0}}},
$$


$S_{p}$ being the exchange surface between the pellet and the oven and $M_{m}$ the molar mass of 2-HP. Note that here at step $0, P_{m}$ is equal to the total pressure $P$ in the oven, since no carrier gas is present. When Eq. 6 will be used below at steps 1 and 2, $P_{m}$ will be the partial pressure of 2-HP in the oven.

As said above, a steady state is created where all the molecules evaporating from the pellet surface leave the oven as an effusive beam through the $D_{2}$ nozzle. Hence, $\Phi_{m, i}=\Phi_{m, o}$. The effusive character of the $\Phi_{m, o}$ flow stems from the fact that the mean free path of the molecule is larger than the nozzle size $\lambda_{0} \gg D_{2}$. As a results, $\Phi_{m, o}$ can be evaluated using Eq. 3 and $\Phi_{m, i}=\Phi_{m, o}=P_{m} \frac{\pi \aleph_{A} D_{2}^{2}}{4 M_{m}} \sqrt{\frac{1}{2 \pi} \frac{M_{m}}{\Re T_{0}}}$. When replacing $\Phi_{m, i}$ by its expression (Eq. 6), $P_{m}$ is obtained. It fixes the pressure condition before the carrier gas starts flowing into the oven when the valve opens at time $t=0$ :

$$
P_{m}(t \leq 0)=\frac{P_{s}}{1+\frac{\pi D_{2}{ }^{2}}{4 S_{p}}} .
$$

Step 1 - Two sequential expansions. This step begins when the valve opens up $(t=0)$ and ends when it closes $(t=\delta \tau)$. The valve opening generates a gas pulse with the carrier gas, which experiences a first expansion from the foreline of the valve (stagnation conditions $P_{0}, T_{0}$ ) into the oven, then a second from the oven into the source chamber (pressure $P_{1}$ ). The carrier gas spends enough time in the oven to thermalize at temperature $T_{0}$ and mixes together with 2-HP molecules. The second expansion thus carries both the carrier gas and 2-HP molecules.

In contrast with step 0 , no steady state is created during step 1 . The partial pressures $P_{c g}$ and $P_{m}$ of the carrier gas and 2-HP molecules and the total pressure $P$ inside the oven $\left(P=P_{c g}+P_{m}\right)$ are therefore time dependent quantities. The flow rates of the carrier gas and 2-HP molecules for entering and leaving the oven are no longer in balance:

$$
\begin{aligned}
& \Delta \Phi_{c g}(t)=\Phi_{c g, i}(t)-\Phi_{c g, o}(t) \neq 0 \\
& \Delta \Phi_{m}(t)=\Phi_{m, i}(t)-\Phi_{m, o}(t) \neq 0
\end{aligned}
$$

where $\Phi_{c g, i}(t)$ (resp $\Phi_{c g, o}(t)$ ) is the flow rate of the carrier gas entering into (resp. leaving) the oven at time $t . \Phi_{m, i}(t)$ and $\Phi_{m, o}(t)$ were defined at step 0 but here, an explicit dependence over $t$ is given.

The 2-HP sublimation through the pellet surface is active during step 1 as it was at step 0 . It maintains a flow rate $\Phi_{m, i}(t)$ of 2-HP molecules entering into the oven. It is given by Eq. 6 as at step 0 but here, $P_{m}$ varies as a function of time because 2-HP is driven out of the oven by the time-dependent flow of the carrier gas.

The stagnation pressure $P_{0}$ of the carrier gas and the pressure ratio $\frac{P_{0}}{P}$ are large enough to ensure that the first expansion is supersonic. The $\Phi_{c g, i}$ flow rate is therefore obtained by writing Eq. 2 for the expansion of a gas of molar mass $M_{c g}$, stagnation pressure $P_{0}$, through a nozzle of diameter $D_{1}$ :

$$
\Phi_{c g, i}(0 \leq t \leq \delta \tau)=P_{0} \frac{\pi}{4} \frac{\aleph_{A} D_{1}^{2} C}{M_{c g}} \sqrt{\frac{M_{c g}}{\Re T_{0}}} .
$$

It is a steady flow rate since the pressure $P_{0}$ in the foreline of the pulsed valve does not vary as a function of time. 
The flow rates $\Phi_{c g, o}$ and $\Phi_{m, o}$ of the carrier gas and 2-HP molecules out of the oven proceed from the expansion through the nozzle of diameter $D_{2}$ (second expansion). The carrier gas/2-HP mixture is in the ratio of the partial pressures $P_{c g}$ and $P_{m}$. At the very beginning of step 1 , when the carrier gas pressure inside the oven has not built up completely, the expansion is effusive. Later, during most of step 1, the expansion becomes supersonic. To account for the early stage of this step and to prepare the treatment of step 2 below, Eq. 4 describing a transition regime is used to calculate the flow rates $\Phi_{c g, o}$ and $\Phi_{m, o}$ out of the oven:

$$
\begin{aligned}
\Phi_{c g, o}=f_{S}\left(K_{n}\right) \cdot \Phi_{\text {super }}\left(P_{c g}\right)+ \\
{\left[1-f_{S}\left(K_{n}\right)\right] \cdot \Phi_{\text {eff }}\left(P_{c g}\right) } \\
\Phi_{m, o}=f_{S}\left(K_{n}\right) \cdot \Phi_{\text {super }}\left(P_{m}\right)+ \\
{\left[1-f_{S}\left(K_{n}\right)\right] \cdot \Phi_{\text {eff }}\left(P_{m}\right) . }
\end{aligned}
$$

The quantities $\Phi_{\text {super }}\left(P_{c g}\right.$ or $\left.P_{m}\right)$ and $\Phi_{e f f}\left(P_{c g}\right.$ or $\left.P_{m}\right)$, which appear in these expression are deduced from Eqs. 2 and 3 for an expansion through a nozzle of diameter $D_{2}$. The Knudsen number is evaluated from the total pressure $P$ and links these two equations.

When including the flow rates $\Phi_{m, i}$ (Eq. 6), $\Phi_{c g, i}$ (Eq. 9), $\Phi_{c g, o}$ and $\Phi_{m, o}$ (Eq. 10) into Eq. 8 , the quantity $\Delta \Phi_{c g, o}$ and $\Delta \Phi_{m, o}$ can be calculated. The conservation of matter and the assumption that both the carrier gas and 2-HP behave as an ideal gas provide the differential equations which control the carrier gas and 2-HP partial pressures:

$$
\begin{aligned}
& \frac{V \aleph_{A}}{\Re T_{0}} \frac{d P_{c g}}{d t}=\Delta \Phi_{c g}(t) \\
& \frac{V \aleph_{A}}{\Re T_{0}} \frac{d P_{m}}{d t}=\Delta \Phi_{m}(t)
\end{aligned}
$$

where $V$ is the volume of the oven. Numerical integration of Eqs. 11 provides us with the flow rates $\Phi_{c g, o}$ and $\Phi_{m, o}$ as a function of time over the interval $0 \leq t \leq \delta \tau$.

Step 2 - Emptying the oven. The pulsed valve is closed and no carrier gas enters any more into the oven $\left(\Phi_{c g, i}=0\right)$. Modeling this step is performed by just turning $P_{0}$ to zero in Eq. 9 and pursuing the integration up to the next valve opening at $\mathrm{t}=50 \mathrm{~ms}$.

\subsection{Velocity distribution of the beam particles}

Gaveau [19] (alternatively see Kantrowitz and Grey, [1]) reports a general expression for the velocity distribution of beam particles which applies both for describing effusive and supersonic beams:

$$
f(v)=F_{N} v^{2} \exp \left(-\frac{M}{2 \Re T}\left[V_{\text {beam }}-v\right]^{2}\right) .
$$

$T$ is the temperature inside the beam. $V_{\text {beam }}$ is the hydrodynamical velocity of the beam particles and $M$ their molar mass. These three parameters are examined be- 
low, whether the expansion is effusive or supersonic. The normalization factor $F_{N}$ is obtained by numerical integration. It insures that $\int_{0}^{\infty} f(v) d v=1$.

Eq. 12 was chosen for its simplicity. It assumes a spherical Maxwellian velocity distribution. Many authors (e.g. Ref.[23]) refined this description by making the distribution ellipsoidal, hence defining two internal temperatures in the beam, one in the beam direction $\left(T_{\|}\right)$and the other, $T_{\perp}$ in the perpendicular direction. Here, $T_{\perp}=T_{\|}=T$ is assumed.

We examine now, how Eq. 12 applies both to effusive and supersonic expansions and how the Knudsen number and switch function $f_{S}\left(K_{n}\right)$ are included very simply into this equation to make it describing the transition between the effusive and supersonic flow regimes.

No stagnation enthalpy is converted as kinetic energy within the expanding gas when the expansion is effusive. Hence, $T=T_{0}, V_{\text {beam }}=0$ and Eq. 12 reproduces the standard velocity distribution of an effusive beam $\left(\propto v^{2} \exp \left(-\frac{M v^{2}}{2 \Re T_{0}}\right)\right)$. In the flow regime provided by an ideal supersonic expansion, the stagnation enthalpy is converted partly as kinetic energy. This leads to a low, final temperature $T=T_{\text {super }}$. $T_{\text {super }}=4 \mathrm{~K}$ is chosen here. The switch function $f_{S}\left(K_{n}\right)$ is used to transform the temperature $T=T_{0}$, which describes the effusive expansion in Eq. 12, into $T=T_{\text {super }}$ for the supersonic expansion. The expression $T\left(K_{n}\right)=T_{0}+\left(T_{\text {super }}-T_{0}\right) f_{S}\left(K_{n}\right)$ is used. The regime-dependent parameter $V_{\text {beam }}\left(K_{n}\right)$ which appears also in Eq. 12 is deduced from $T\left(K_{n}\right)$ by the conservation of energy. Accordingly:

$$
\frac{5}{2} \Re T_{0}=\frac{5}{2} \Re T\left(K_{n}\right)+\frac{1}{2} M V_{\text {beam }}\left(K_{n}\right)^{2} .
$$

The latter expression reflects the essence of a supersonic expansion which converts part of the molar enthalpy $\frac{5}{2} \Re T_{0}$ of the gas under stagnation conditions into kinetic energy $\frac{1}{2} M V_{\text {beam }}^{2}$. It assumes implicitly that only the translational energy of the gas participates to the energy conversion. This is correct for the rare gas carrier, but assumes that the vibrational and rotational energy of the seeded molecules (the 2-HP molecules) do not participate to the expansion.

The molar mass $M$ is the last parameter which appears in Eq. 12 and depends on the flow regime. It is equal to the molar mass of the expanding gas when the expansion is effusive (either $M_{c g}$ for the carrier gas or $M_{m}$ for the 2-HP molecule. Note that the two gases expand independently under this regime, which is collisionless), whereas it is equal to the average molar mass $M_{c g} \frac{P_{c g}}{P}+M_{m} \frac{P_{m}}{P}$ in an ideal supersonic expansion. Accordingly:

$$
\begin{array}{r}
M_{c g}\left(K_{n}\right)=\left(M_{c g} \frac{P_{c g}}{P}+M_{m} \frac{P_{m}}{P}\right) \cdot f_{S}\left(K_{n}\right)+ \\
M_{c g} \cdot\left[1-f_{S}\left(K_{n}\right)\right] \\
M_{m}\left(K_{n}\right)=\left(M_{c g} \frac{P_{c g}}{P}+M_{m} \frac{P_{m}}{P}\right) \cdot f_{S}\left(K_{n}\right)+ \\
M_{m} \cdot\left[1-f_{S}\left(K_{n}\right)\right] .
\end{array}
$$

This expression allows the velocity distribution of the seeded molecules to slip with respect to that of the carrier gas during the transition regime.

After including explicitly the dependence of $M, T$ and $V_{\text {beam }}$ on the Knudsen num- 
ber, Eq. 12 becomes:

$$
f\left(v, K_{n}\right)=F_{N} v^{2} \times \quad \quad \exp \left(-\frac{M(K n)}{2 \Re T\left(K_{n}\right)}\left[V_{\text {beam }}\left(K_{n}\right)-v\right]^{2}\right) .
$$

\subsection{Gas flow entering into the detection zone}

The molecular beam is skimmed before interaction with the lasers. The detection region therefore intercepts a fairly small solid angle $\Delta \Omega$ along the beam axis and the detected flow rate, either $\Phi_{\text {super }}^{\Delta \Omega}$ or $\Phi_{\text {eff }}^{\Delta \Omega}$ whether a supersonic or an effusive regime is at play, is close to the centerline flow rate. The proportionality factor between these quantities and the total flow rates $\Phi_{\text {super }}$ and $\Phi_{\text {eff }}$ that appear in Eq. 10 depends on the flow regime since supersonic expansions are more directive than effusive ones.

Beijerinck and Verster proposed a general formula to account for the angular distribution of beam intensities in both supersonic and effusive expansions. [24] Equation 12 and Table I of Ref. [24] propose an angular dependence as $\cos ^{b} \theta$ where $b=1$ describes an effusive expansion and $b>1$ a supersonic one. The larger the $b$ value, the larger the directivity of the expansion. Beijerinck and Verster[24], proposed that $b=3$ adequately describes the supersonic expansion of a monoatomic gas. Since 2-HP is a minor component of the beam under the supersonic regime, we consider that the angular distribution is governed by the monoatomic carrier gas. The value $b=3$ is used for both the carrier gas and the 2-HP molecule under this flow regime.

Let $\Delta \theta$ be the opening angle of the detection zone along the beam axis and $\phi$ the azimuthal angle about this axis. Assuming that the expansion fills the half space at the oven outlet ( $D_{2}$ nozzle) and has the cylindrical symmetry, $\Delta \Omega=\pi \Delta \theta^{2}$ and $\frac{\int_{0}^{2 \pi} d \phi \int_{0}^{\Delta \theta} \cos ^{b} \theta \sin \theta d \theta}{\int_{0}^{2 \pi} d \phi \int_{0}^{\frac{\pi}{2}} \cos ^{b} \theta \sin \theta d \theta}$ for $b=3$ is the proportionality factor between $\Phi_{\text {super }}^{\Delta \Omega}$ (resp. $b=1$ and $\Phi_{\text {eff }}^{\Delta \Omega}$ ) and $\Phi_{\text {super }}\left(\right.$ resp. $\Phi_{\text {eff }}$ ). For small values of $\Delta \theta$ as considered here, the proportionality factor becomes $2 \Delta \theta^{2}$ for the supersonic flow regime and $\Delta \theta^{2}$ for the effusive one. Hence,

$$
\begin{aligned}
\Phi_{\text {super }}^{\Delta \Omega}\left(P_{i}\right) & =\Phi_{\text {super }}\left(P_{i}\right) \times \frac{2}{\pi} \Delta \Omega \\
\Phi_{\text {eff }}^{\Delta \Omega}\left(P_{i}\right) & =\Phi_{\text {eff }}\left(P_{i}\right) \times \frac{1}{\pi} \Delta \Omega
\end{aligned}
$$

where $P_{i}$ is the partial pressure of the gas under consideration.

With these expressions, Eq. 10 allows us calculating the relevant flow rates at the detection region as:

$$
\begin{aligned}
\Phi_{c g, d e t}=f_{S}\left(K_{n}\right) \Phi_{\text {super }}\left(P_{c g}\right) \times \frac{2}{\pi} \Delta \Omega+ \\
{\left[1-f_{S}\left(K_{n}\right)\right] \Phi_{e f f}\left(P_{c g}\right) \times \frac{1}{\pi} \Delta \Omega } \\
\Phi_{m, \text { det }}=f_{S}\left(K_{n}\right) \Phi_{\text {super }}\left(P_{m}\right) \times \frac{2}{\pi} \Delta \Omega+ \\
{\left[1-f_{S}\left(K_{n}\right)\right] \Phi_{\text {eff }}\left(P_{m}\right) \times \frac{1}{\pi} \Delta \Omega . }
\end{aligned}
$$


When writing the above expression, it has been implicitly assumed that when it passes through the nozzle of diameter $D_{2}$, the gas flow reaches very quickly a free molecular regime. As a result, no collision affects the velocity distribution and the flow rate of the carrier gas and of the 2-HP molecules over the distance $R$ between this nozzle and the interaction region with the laser. Accordingly, the numerical density $N_{c g, d e t}$ of the carrier gas atoms in the detection region is given by

$$
\begin{aligned}
N_{c g, \text { det }}(t)=\int_{0}^{+\infty} f\left(v, K_{n}\left[P\left(t-\frac{R}{v}\right)\right]\right) \times & \\
& \frac{\Phi_{c g, d e t}\left(t-\frac{R}{v}\right)}{v} \frac{1}{4 \pi R^{2}} d v .
\end{aligned}
$$

A similar expression holds for the 2-HP molecules when replacing the subscript $c g$ by $m$. The simulated quantities $N_{c g, \text { det }}(t)$ and $N_{m, \text { det }}(t)$ are those to be compared to the experimental results. The Knudsen number which appears in these expressions is written $K_{n}\left[P\left(t-\frac{R}{v}\right)\right]$ to make clear that it depends explicitly on the pressure in the oven at time $\left(t-\frac{R}{v}\right)$.

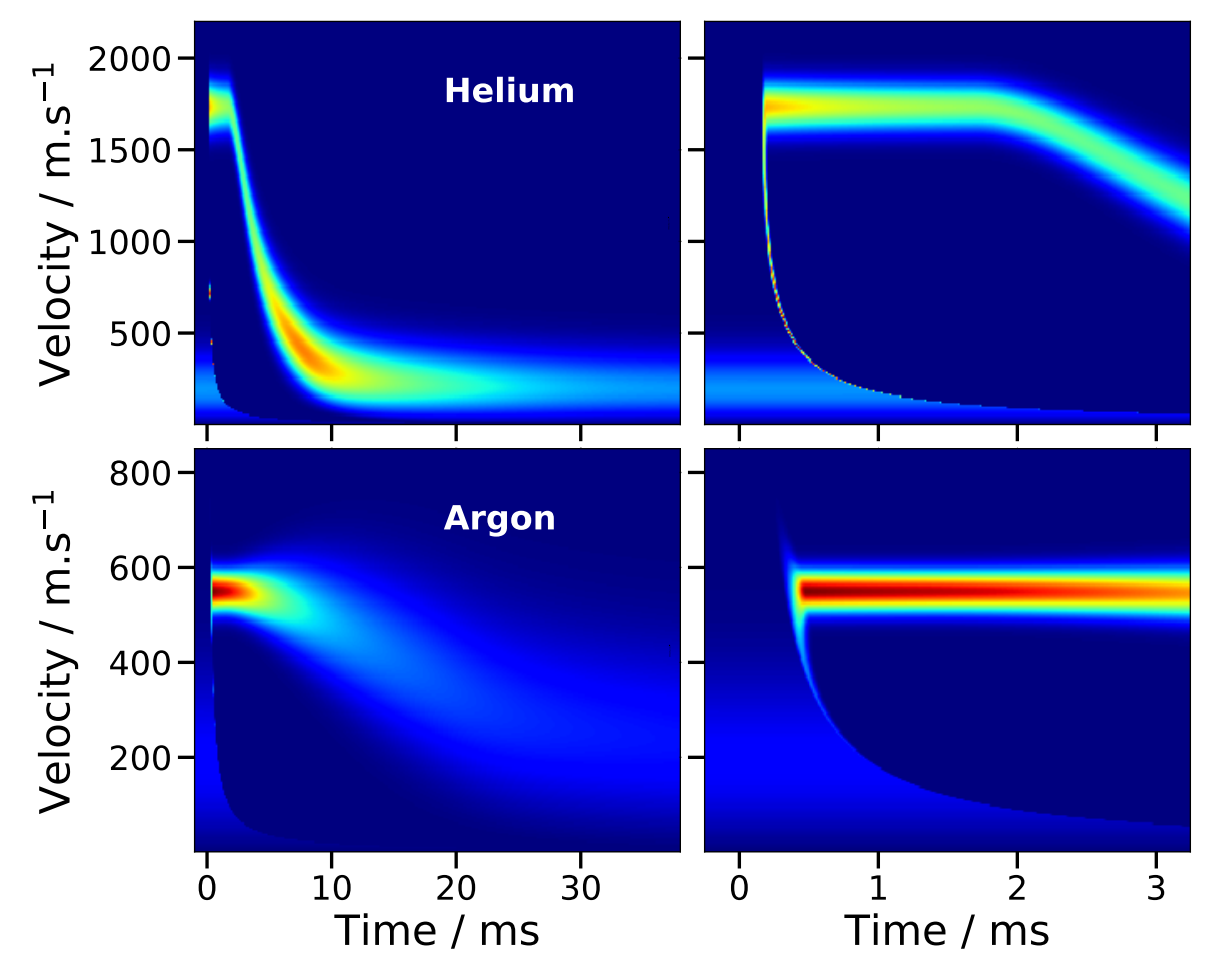

Figure 8. Same caption as Fig. 4. Here, the simulated 2-HP number density is displayed instead of the measured photoion intensity. The color scale is the same than that of Fig. 4.

\section{Simulation results}

The above simulation model allows us calculating the number density and the velocity distribution of the 2-HP molecules in the detection region of the experiment. The values of the parameters used for the simulation are listed in appendix A. The simulation 

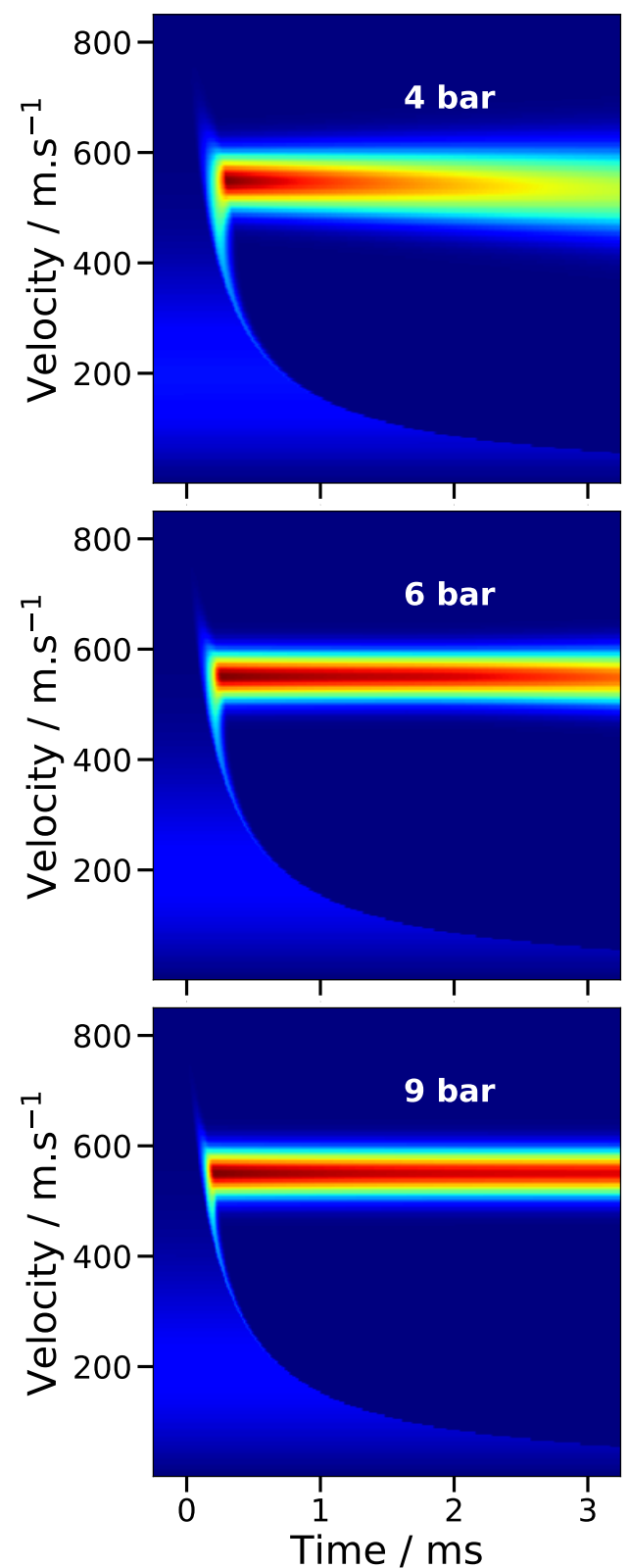

Figure 9. Same caption as Fig. 5 for the simulated number density of $2 \mathrm{HP}$ in the detection zone instead of the measured ion signal of 2-HP. The color scale is the same than that of Fig. 4. 
results corresponding to a $P_{0}=6$ bar stagnation pressure of either helium or argon as carrier gas are shown in Fig. 8 with scales that allows direct comparison with the experimental results of Fig. 4. In the same way, Fig. 9 presents simulation results that can be compared directly to the experimental data of Fig. 5 where argon is the carrier gas and three stagnation pressures are sampled.

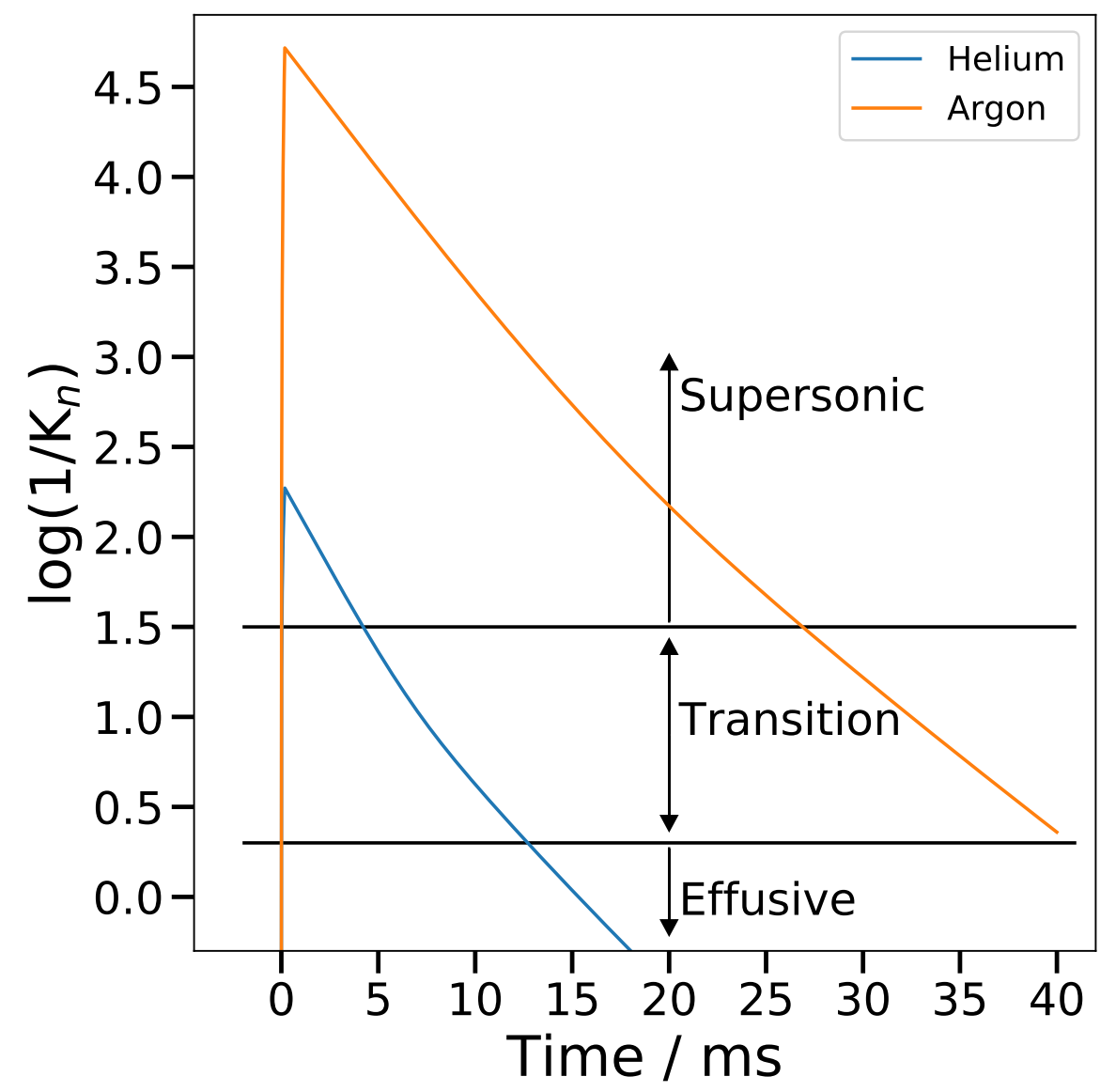

Figure 10. Simulated Knudsen number $\left(\log \left(1 / K_{n}\right)\right.$ is plotted) as a function of the time delay after the valve opening.

Finally, to help the discussion, Fig.10 shows the Knudsen number in the oven as a function of the time delay after the valve opening. Note that $\log \left(1 / K_{n}\right)$ is plotted instead of $K_{n}$.

\section{Discussion}

The simulation model which is presented above is actually very simple. It is based on a continuum representation of the supersonic expansion and assumes that the pressure is uniform within the oven, immediately after the valve opening. Nevertheless, the qualitative agreement between the simulation (Figs. 8 and 9) and the experiment (Figs. 4 and 5) is striking. The three regions that were distinguished in Sec. 3 (Experimental Results) can be recognized indeed in Figs. 8 and 9: 
The small velocity region - The color code and the scales in the first row of Fig. 8 (helium data) makes especially apparent the velocity class centred about $200 \mathrm{~m} . \mathrm{s}^{-1}$. It is present at early time and reappears after about $20 \mathrm{~ms}$ as observed experimentally. The color code in the second row of this figure (argon data) makes this velocity class less visible, although existing.

The intermediate region - The complex region between 0.3 and $0.5 \mathrm{~ms}$ in helium (0.5-0.7 $\mathrm{ms}$ in argon) where the velocities change suddenly also appears in the simulation. However, only two classes of velocities are present within this time window when three are observed in the experiment. This is especially apparent in the argon data at three different stagnation pressures (compare Fig. 5 with Fig. 9). Moreover the time gap, which is apparent in the experimental data between the slow and rapid velocity components, does not exist in the simulation.

The decreasing velocity region - The extended region where the velocity decreases from $1600 \mathrm{~m} . \mathrm{s}^{-1}$ at $\approx 0.5 \mathrm{~ms}$ in helium (resp. $550 \mathrm{~m} . \mathrm{s}^{-1}$ at $\approx 1 \mathrm{~ms}$ in argon) down to about $200 \mathrm{~m} . \mathrm{s}^{-1}$ is also predicted by the simulation. However, the simulated decrease is slower than observed experimentally. Also, the intensity ratio between the rapid and the slow velocity classes is overestimated by the simulation.

This overall agreement between simulations and experiment suggests that the present model has caught key aspects of the gas flows inside the beam source. Of course not all of them, since a velocity component is missing in the intermediate region, which has moreover a more complex structure than predicted. This deserves a more elaborate discussion.

Changes in the flow regime, whether it is effusive, supersonic or a transition one has been anticipated several times in the present paper. This can be stated on a quantitative basis using the variation of the Knudsen number reported in Fig. 10. Before the valve opening and after $10 \mathrm{~ms}$ in helium (26 ms in argon) the flow regime is effusive. It is supersonic during a short time after the valve opening: $5 \mathrm{~ms}$ in helium and $10 \mathrm{~ms}$ in argon. Between these limits, a transition regime is at play.

It is well known that in a supersonic expansion, the beam particles have a much larger average velocity than in an effusive expansion (see Eq. 15 whether $v_{\text {beam }} \neq 0$ or $\left.v_{\text {beam }}=0\right)$. In line with the discussion of the experimental results in Sec. 4, the shape of Fig. 10 for the Knudsen number (actually $\log \left(1 / K_{n}\right)$ ) guides the scheme in Fig. 11 where the flow regime is put in correspondance with the velocity distribution as a function of time.

We already noticed the strong resemblance between experiment and simulation either when comparing Fig. 4 (experiment at 6 bar) and Fig. 8 (corresponding simulation) or when observing Fig. 9 where experimental and simulation results are reported for the argon expansion at 4, 6 and 9 bar. However, a point is never reproduced by the simulation when argon is the carrier gas: the narrow feature which extends about a delay time of $0.5 \mathrm{~ms}$ between a 400 to $800 \mathrm{~m} . \mathrm{s}^{-1}$ velocity. From Fig. 4 we know that this feature is synchronized with the valve opening since it follows the lower white curve.

We already mentioned that the present simulation model is based on a continuum representation of the supersonic expansion and assumes that the pressure is uniform within the oven. It bypasses any non-uniform behaviours that may exist when expanding carrier gas atoms of large velocity collide with thermal 2-HP molecules in the oven. In other words, the valve opening induces a high pressure flow into the the oven, which 


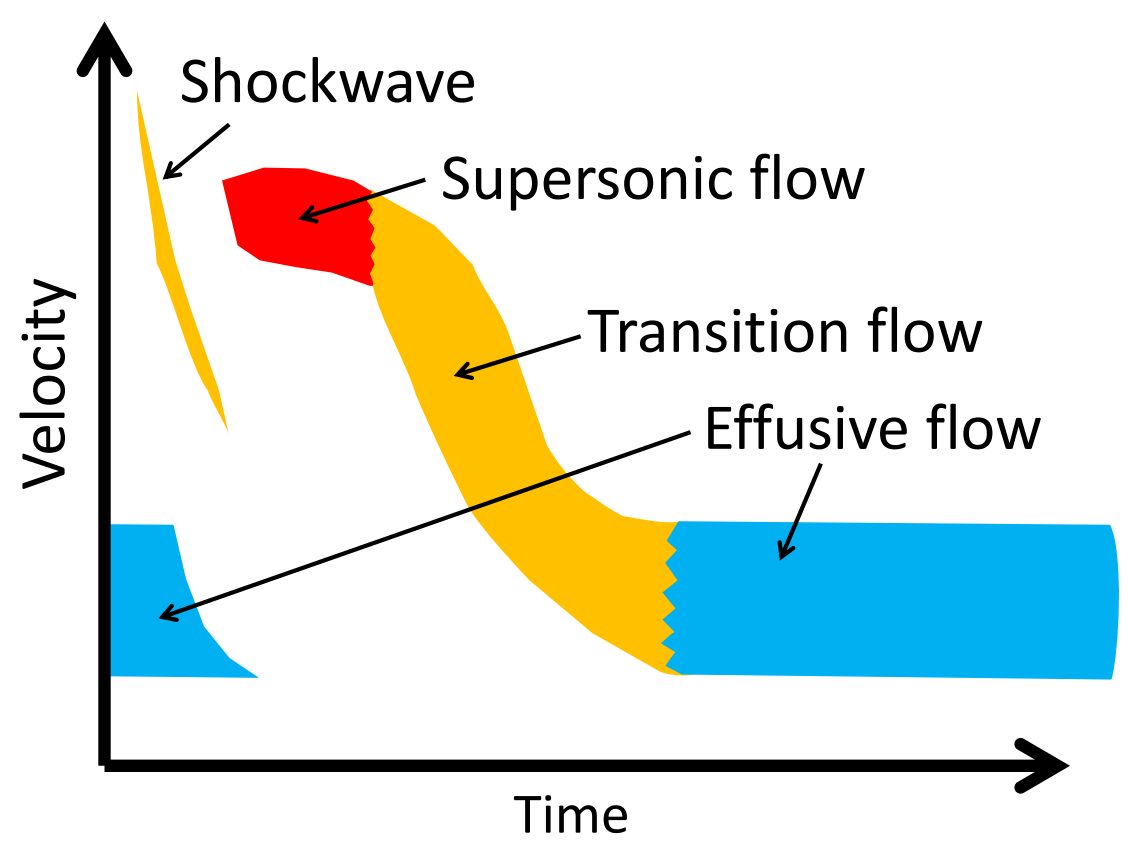

Figure 11. Scheme showing the correspondance between the flow regime and the velocity distribution as a function of time.

may develop a shockwave. Such phenomenon is not taken into account by the present simulation. Given the otherwise nice agreement between experiment and simulation, we consider that the non-simulated feature is associated with the development of such a shockwave. This leads to a sudden large increase of the temperature, velocity, density and pressure inside the oven and subsequently to a dramatic change in the free expansion from the oven, as observed experimentally.

This scenario mimics on a small scale unsteady behaviours observed in shock tubes, for instance the unsteady shock propagation observed by Stalker and Mudford, in a steady flow nozzle expansion.[25] These authors observed that the axial density distribution associated with the prior steady flow allows the unsteady flow following the nozzle primary starting shock to accelerate from supersonic to hypersonic speeds.

A similar observation seems to be at play here with argon as carrier gas since the largest velocities in the feature associated with the shockwave $\left(c a .850 \mathrm{~m} . \mathrm{s}^{-1}\right)$ exceeds significantly that observed when the expansion is purely supersonic $\left(c a .4-500 \mathrm{~m} . \mathrm{s}^{-1}\right)$ (see Figs. 4 and 5).

A last point must be made concerning the shockwave signal in Fig. 5). Its time extension is shorter when the pressure increases from 4 to 9 bar and its velocity distribution shifts to higher velocities. This suggests that larger stagnation pressures in the foreline of the pulsed valve favour the construction of a stronger shockwave in a shorter time.

At longer time when the shockwave sequence is over, the close ressemblance between the experimental and the simulated velocity distributions suggests that the gas flow follows the sequence suggested by Fig. 10: a supersonic $\rightarrow$ transition $\rightarrow$ effusive flow. When observing the lower panels in Fig. 4, this corresponds to the ca. $400 \mathrm{~m} . \mathrm{s}^{-1}$ average velocities at a $t=0.7 \mathrm{~ms}$ delay time which slowly decrease as $t$ increases. This 
was discussed in Sec. 4 on a purely experimental basis. It is confirmed here by the simulation.

Finally, Fig. 9 show results at 4, 6 and 9 bar for the argon expansion. As expected and as observed experimentally in Fig. 5), increasing the stagnation pressures are associated with an improved quality of the supersonic expansion, i.e. a better conversion of the stagnation enthalpy into kinetic energy and a smaller velocity slip between argon and 2-HP. This figure shows indeed that the average velocity of the 2-HP molecules (taken at a $0.7 \mathrm{~ms}$ time delay) moves up from $\approx 450 \mathrm{~m} \cdot \mathrm{s}^{-1}$ at 4 bar to $\approx 550 \mathrm{~m} \cdot \mathrm{s}^{-1}$ at 9 bar.

\section{Conclusion}

The present paper reports experimental and simulation results concerning the velocity distribution of 2-hydroxypyridine (2-HP) molecules in a seeded pulsed beam where the carrier gas is either helium or argon. Experimentally, the beam is generated in a two stage expansion, where the first stage is driven by a pulsed valve. Then, standard techniques used in real-time reaction dynamics experiments, namely photoionization by a pulsed laser and velocity imaging, allows us documenting the variation of the velocity distribution of 2 - $\mathrm{HP}$ as a function of time, after the valve opening.

On the simulation side, rather than seeking an extensive treatment, the idea is to identify the various flow regime that are at play: the pure free molecular ( or effusive) flow regime, the transition flow regime, and the continuum (or supersonic) flow regime. The motivation is to identify features in the observed phenomena which canot be assigned to a pure supersonic or a pure effusive flow regime. Two are identified. One is assigned to the transition regime between supersonic and effusive flow regimes after the valve closes. The latter was simulated quantitatively using an empirical description of the switch between supersonic and effusive flow regimes. The second one is an unsteady flow regime where a shockwave is developing in the intermediate chamber between the two stages of the expansion. This shockwave builds the conditions of a supersonic flow in the second stage of the expansion.

Our hope is that the present experimental technique offers a novel material to support recent developments in mathematics which aim at modeling gas flows in regimes, covering continuum, slip, transition and free molecular flows. The two phenomena that have just been mentioned may appear as ideal for such testing. The present experimental technique offers a rare opportunity to observe the velocity distributions of the gas particles as a shockwave is formed and propagates and later on, as a supersonic flow becomes slowly effusive. The latter case is especially interesting since the slow return to the effusive flow regime almost necessarily involves a velocity slip between the carrier gas and the seeded molecules. Again, the possibilities offered by the present experimental technique may appear as a stringent test when modeling such a slip.

\section{Acknowledgments}

The authors kindly thank David Parker and André Eppink for their help in building our VMI. The authors thank D. Guillaumet, M. Perdrix and Dr. O. Gobert for running the SLIC/LUCA laser facility. Financial support is acknowledged from the Agence Nationale pour la Recherche (ANR-14-CE32-0010 XTASE). 


\section{References}

[1] A. Kantrowitz and J. Grey, Rev. Sci. Instrum. 22, 328-332 (1951).

[2] R. Campargue, J. Phys. Chem. 88, 4466-4474 (1984).

[3] J.M. Hayes, Chem. Rev. 87, 745-760 (1987).

[4] G. Scoles, Atomic and Molecular Beam Methods (, , 1988).

[5] H.J. Saleh and A.J. McCaffery, J. Chem. Soc. Faraday Trans. 89, 3217-3221 (1993).

[6] V. Aquilanti, M. Bartolomei, F. Pirani, D. Cappelletti, F. Vecchiocattivi, Y. Shimizu and T. Kasai, Phys. Chem. Chem. Phys. 7, 291-300 (2005).

[7] A. Lietard, L. Poisson, J.M. Mestdagh and M.A. Gaveau, AIP Conf. Proc. 1786, 060004 (2016).

[8] G. A. Bird, The Physics of Fluids 6, 1518 (1963).

G. A. Bird, The DSMC Method, Version 1.2, CreateSpace Independent Publishing Platform ed. (2013).

[9] A.T.J.B. Eppink and D.H. Parker, Rev. Sci. Instrum. 68 (9), 3477-3484 (1997).

[10] L. Poisson, K.D. Raffael, B. Soep, J.M. Mestdagh and G. Buntinx, J. Am. Chem. Soc. 128 (10), 3169-3178 (2006).

[11] L. Poisson, R. Maksimenska, B. Soep, J.M. Mestdagh, D.H. Parker, M. Nsangou and M. Hochlaf, J. Phys. Chem. A 114 (9), 3313-3319 (2010).

[12] C. Abeysekera, B. Joalland, Y. Shi, A. Kamasah, J.M. Oldham and A.G. Suits, Rev. Sci. Instrum. 85 (2014).

[13] N. Shafizadeh, L. Krim, S. Sorgues and B. Soep, Chem. Phys. Lett. 357, 37-44 (2002).

[14] MOLBASE Encyclopedia https://www.molbase.com/moldata/ 2019.

[15] J.C. Poully, J.P. Schermann, N. Nieuwjaer, F. Lecomte, G. Gregoire, C. Desfrancois, G.A. Garcia, L. Nahon, D. Nandi, L. Poisson and M. Hochlaf, Phys. Chem. Chem. Phys. 12 (14), 3566-3572 (2010).

[16] L. Poisson, D. Nandi, B. Soep, M. Hochlaf, M. Boggio-Pasqua and J.-M. Mestdagh Phys. Chem. Chem. Phys. 16, 581 (2014).

[17] A.V. Baklanov, L.M.C. Janssen, D.H. Parker, L. Poisson, B. Soep, J.M. Mestdagh and O. Gobert, J. Chem. Phys. 129 (2008).

[18] Z.H. Li, A.P. Peng, H.X. Zhang and J.Y. Yang, Progress in Aerospace Sciences 74, 81-113 (2015).

[19] M.A. Gaveau, Ph. D. thesis, Université Paris-Sud, 1984.

[20] G. Scoles, Atomic and Molecular Beam Methods, Vol. 1 (, , 1988).

[21] J. Vogt and S. Alvarez, Inorg. Chem. 53, 9260-9266 (2014).

[22] J. Barrett and C. Clement, J. Colloid Interface Sci. 150 (2), 352-364 (1992).

[23] A.S. Palau, S.D. Eder, T. Andersen, A.K. Ravn, G. Bracco and B. Holst, Phys. Rev. A 98, 063611 (2018).

[24] H.C.W. Beijerinck and N.F. Verster, Physica B+C 111, 327-352 (1981).

[25] R.J. Stalker and N.R. Mudford, Journal of Fluid Mechanics 241, 525-548 (1992).

\section{Appendix A.}


Table A1. Notations

\begin{tabular}{|c|c|c|}
\hline Quantity & Notation & Value \\
\hline \multicolumn{3}{|l|}{ Fundamental constants } \\
\hline Ideal gas constant & $\Re$ & $8.314 \mathrm{~J} . \mathrm{K}^{-1} \cdot \mathrm{mol}^{-1}$ \\
\hline Avogadro number & $\aleph_{A}$ & $6.02210^{23}$ \\
\hline \multicolumn{3}{|l|}{ Operating conditions } \\
\hline Opening time of the pulsed valve & $\delta \tau$ & 180 or $190 \mu \mathrm{s}$ \\
\hline Period of the pulsed valve & $T_{r}$ & $50 \mathrm{~ms}$ \\
\hline Source temperature & $T_{0}$ & $293.0 \mathrm{~K}$ \\
\hline Pressure in the foreline of the pulsed valve & $P_{0}$ & 5,6 and 9 bar \\
\hline Pressure in the source chamber & $P_{1}$ & $\sim 10^{-5}$ mbar \\
\hline \multicolumn{3}{|l|}{ Physical properties of the beam particles } \\
\hline Molar mass of the carrier gas & $M_{c g}$ & $\mathrm{He}\left(4.00\right.$ g. $\left.\mathrm{mol}^{-1}\right)$ or $\mathrm{Ar}\left(39.95\right.$ g. $\left.\mathrm{mol}^{-1}\right)$ \\
\hline Molar mass of 2-Hydroxypyridine (2-HP) & $M_{m}$ & 95.10 g. $\mathrm{mol}^{-1}$ \\
\hline Van der Waals radius of the carrier gas atoms & $R_{v d w}$ & $\mathrm{He}(0.143 \mathrm{~nm})$ or $\mathrm{Ar}(0.194 \mathrm{~nm})[21]$ \\
\hline Saturation vapor pressure of 2 - $\mathrm{HP}$ at temperature $T_{0}$ & $P_{s}$ & $3.410^{-4} \mathrm{mbar}$ \\
\hline \multicolumn{3}{|l|}{ Source geometry } \\
\hline Nozzle diameter of the pulsed valve & $D_{1}$ & $300 \mu \mathrm{m}$ \\
\hline Nozzle diameter of the oven & $D_{2}$ & $2.0 \mathrm{~mm}$ \\
\hline Skimmer diameter & $d_{e}$ & $1.0 \mathrm{~mm}$ \\
\hline Distance between the nozzles $D_{1}$ and $D_{2}$ & $R_{0}$ & $1.83 \mathrm{~cm}$ \\
\hline Distance between the $D_{2}$ nozzle and the skimmer & $l$ & $5.0 \mathrm{~cm}$ \\
\hline Distance between the nozzle $D_{2}$ and the detection zone & $R$ & $17.5 \mathrm{~cm}$ \\
\hline Exchange surface between the pellet and the oven chamber & $S_{p}$ & $4.74 .10^{-5} \mathrm{~m}^{2}$ \\
\hline Volume of the oven & $V$ & $4.0 \mathrm{~cm}^{3}$ \\
\hline
\end{tabular}

\section{Quantities used to describe the transition flow regime}

Knudsen number Switch function between the free molecular and continuum flow regimes Average molar mass of the gas filling the oven Average heat capacity ratio of the gas filling the oven Velocity distribution of the beam particles

$$
\begin{aligned}
& K_{n} \\
& f_{S}\left(K_{n}\right) \quad \text { Eq. } 1 \\
& M \\
& \gamma=\frac{C_{p}}{C_{v}} \\
& f\left(v, K_{n}\right) \quad \text { Eq. } 12
\end{aligned}
$$

\section{Simulated quantities as a function of time $t$}

Time delay between the valve opening and the laser pulse Total pressure inside the oven Partial pressure of carrier gas in the oven Partial pressure of 2-HP in the oven

Flow rate of 2-HP molecules entering the oven from the pellet Flow rate of 2-HP molecules leaving the oven through the $D_{2}$ nozzle Flow rate of 2 -HP molecules in the detection region Flow rate of carrier gas atoms entering the oven through the $D_{1}$ nozzle 


\section{Graphical abstract}

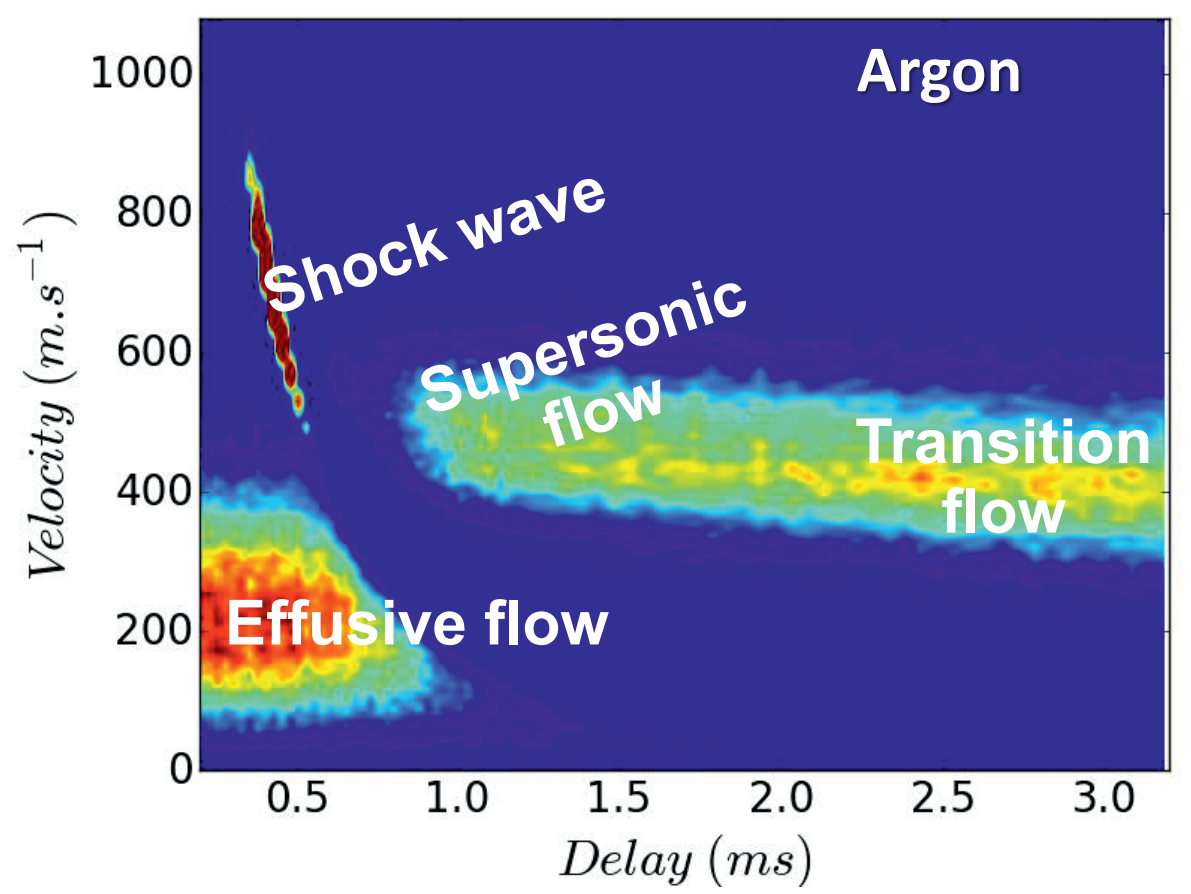

Figure A1. The time-dependent velocity distribution of 2-hydroxypyridine molecules seeded in a pulsed expansion of the carrier gas (helium or argon) is investigated both experimentally and computationally. An interesting shock wave feature is observed a short time after the valve opening when the flow regime switches from effusive to supersonic. 